\title{
The Impact of Meteorological Factors and Air Pollution on the Spread of COVID-19
}

\author{
Danhua Huang ${ }^{1}$, Jiashu Zhao ${ }^{1}$, Yukang Jiang ${ }^{1}$, Ting Tian ${ }^{1}$, Yewei Li $^{2}$, and Xueqin \\ $\mathrm{WANG}^{* 3}$ \\ ${ }^{1}$ School of Mathematics, Sun Yat-sen University, Guangzhou, China \\ ${ }^{2}$ School of Statistics, Capital University of Economics and Business, Beijing, China \\ ${ }^{3}$ School of Management, University of Science and Technology of China, Heifei, China
}

\begin{abstract}
The new coronavirus disease (COVID-19), as a new infectious disease, has relatively strong ability to spread from person to person. This paper studies several meteorological factors and air quality indicators between Shenzhen and Wenzhou, China, and conducts modelling analysis on whether the transmission of COVID-19 is affected by atmosphere. A comparative assessment is made on the characteristics of meteorological factors and air quality in these two typical cities in China and their impacts on the spread of COVID-19. The article uses meteorological data and air quality data, including 7 variables: daily average temperature, daily average relative humidity, daily average wind speed, nitrogen dioxide $\left(\mathrm{NO}_{2}\right)$, atmospheric fine particulate matter $\left(\mathrm{PM}_{2.5}\right)$, carbon monoxide $(\mathrm{CO})$ and ozone $\left(\mathrm{O}_{3}\right)$, a distributed lag non-linear model (DLNM) is constructed to explore the correlation between atmospheric conditions and non-imported confirmed cases of COVID-19, and the relative risk is introduced to measure the lagging effects of meteorological factors and air pollution on the number of non-imported confirmed cases. Our finding indicates that there is significant differences in the relationship between 7 predictors and the transmission of COVID-19 in Shenzhen and Wenzhou. However, all predictors between the two cities have a non-linear relationship with the number of non-imported confirmed cases. The lower daily average temperature has increased the risk of epidemic transmission in the two cities. As the temperature rises, the risk of epidemic transmission in both cities will significantly decrease. The average daily relative humidity has no significant effects on the epidemic situation in Shenzhen, but the lower relative humidity reduces the risk of epidemic spread in Wenzhou. In contrast, meteorological data have significant impacts on the spread of COVID-19 in Wenzhou. The four predictors $\left(\mathrm{NO}_{2}, \mathrm{PM}_{2.5}, \mathrm{CO}\right.$, and $\left.\mathrm{O}_{3}\right)$ have significant effects on the number of nonimported confirmed cases. Among them, $\mathrm{PM}_{2.5}$ has a significant positive correlation with the number of non-imported confirmed cases. Daily average wind speed, $\mathrm{NO}_{2}$ and $\mathrm{O}_{3}$ have different effects on the number of non-imported confirmed cases in different cities.
\end{abstract}

Keywords air quality; distributed lag non-linear model

\footnotetext{
${ }^{*}$ Corresponding author. Email: hawkingwang@gmail.com.
} 


\title{
气象因素及空气污染因素对新型冠状病毒传播的影响探究
}

\author{
黄丹华 ${ }^{1}$, 赵嘉澍 ${ }^{2}$, 蒋宇康 ${ }^{3}$, 田婷 ${ }^{4}$, 李烨薇 ${ }^{5}$, 王学钦 ${ }^{* 6}$ \\ $1,2,3,4,5$ 中山大学数学学院 \\ 6 首都经贸大学统计学院 \\ 6 中国科学技术大学管理学院
}

\begin{abstract}
摘要
新型冠状病毒疾病（COVID-19）作为一种新发传染病，具有较强的人际传播能力。本文研究了 中国深圳市和温州市 2 个城市的若干气象因素与空气质量指标，对 COVID-19 是否受空气影响 进行建模分析。对中国两个典型城市中的气象因素和空气质量的特征及其对 COVID-19 的影响, 进行对比评估。本文利用气象数据与空气质量数据, 包括日平均温度、日平均相对湿度、日平均 风速、二氧化氮 $\left(\mathrm{NO}_{2}\right)$ 、大气细颗粒物 $\left(\mathrm{PM}_{2.5}\right)$ 、一氧化碳 $(\mathrm{CO})$ 和臭氧 $\left(\mathrm{O}_{3}\right)$, 针对 COVID-19 确诊人数构建分布滞后非线性模型（Distributed Lag Non-Linear Model, DLNM），引入了相对 危险度来衡量气象因素和大气污染因素对确诊人数的滞后影响, 并考虑气象因素和大气污染因 素对 COVID-19 发病可能存在滞后效应，探究空气因素与确诊病例的相关关系和趋势变化。文 章发现, 深圳市和温州市在 7 个指标与 COVID-19 的效应关系上呈现明显的差异。但所有指标 在两个城市间, 都与 COVID-19 的患病人数呈现非线性关系。较低的日平均温度使两座城市的 疫情传播的风险有所上升，随着温度的升高，两座城市的疫情传播风险均会显著下降。日平均相 对湿度对深圳疫情情况则几乎没有影响, 而较低的相对湿度使得温州市疫情传播风险降低。相比 而言, 气象数据对温州市的 COVID-19 的传播具有显著影响。 $\mathrm{NO}_{2} 、 \mathrm{PM}_{2.5} 、 \mathrm{CO} 、 \mathrm{O}_{3}$ 四个指标 与 COVID-19 的患病人数存在显著效应。其中 $\mathrm{PM}_{2.5}$ 与 COVID-19 患病人数呈显著正相关关 系; 日平均风速、 $\mathrm{NO}_{2}$ 与 $\mathrm{O}_{3}$ 在不同城市对患病人数的影响有所差异。
\end{abstract}

关键词 分布滞后非线性模型; 空气质量

\section{1 引言}

2019 新型冠状病毒（SARS-CoV-2）引起的疾病是一种新发现的呼吸道传染病（COVID19)，具有较强的人际传播能力，目前在世界范围内超过 200 个国家及地区均造成了严重的影响 (Sohrabi et al., 2020)。虽然该病的病因至今尚未完全明确, 但通过现有的病例分析证实, 新型冠 状病毒主要的传播途径还是呼吸道飞沫传播和接触传播 (靳英辉等, 2020)。通过流行病学调查显 示, 病例多可以追踪到与确诊的病例有过近距离密切接触的情况。在人群接触过程中, 含有病原 体的飞沫受气候因素和空气质量的影响, 可能使接触中的人群受到感染。因而, 气候因素与空气 质量可能从病原体、媒介、传播途径和人体免疫力等方面直接或间接影响传染病的发病趋势。

有关研究的结论表明, 特定的气象条件对呼吸道疾病有一定的诱发作用 (李青春等, 1999)。 这是因为某些气象因素（例如气温、湿度）不仅对生物性病原体的繁殖和传播直接产生影响, 而

*通讯作者。电子邮箱：hawkingwang@gmail.com。 
且可以影响易感人群的抵抗力。王铮等人的研究认为, 严重急性呼吸系统综合征 (Severe Acute Respiratory Syndrome, SARS) 日增人数与气温、湿度等因素的变化有关 (王铮等, 2003)。2019 新型冠状病毒（SARS-CoV-2），与 2002 年出现的 SARS 病毒（SARS-CoV）都属于冠状病毒 家族，且传播渠道相似。王茂等将 COVID-19 疫情与气候结合，收集了 1 月 20 日至 2 月 4 日、 429 个城市和地区每日确诊人数与温度数据, 发现气温与 COVID-19 的传播存在相关性：当平 均气温上升到 8.72 摄氏度时, 日累计确诊人数达到峰值 (Wang et al., 2020)。

另一方面，已有研究表明，空气污染对 COVID-19 的传播有显著关系 (Martelletti et al., 2020; Tian et al., 2020; Yongjian et al., 2020)，例如大气污染颗粒物（atmospheric particulate matter，PM）能够使病毒在空气中存活几小时甚至几天。在一些发达地区，高浓度的 PM 会增 加由于呼吸疾病或心血管疾病引起的死亡 (Ciencewicki et al., 2007)。而二氧化氮 $\left(\mathrm{NO}_{2}\right)$ 作为人 类工业化活动的追踪指标，例如汽车尾气。它的浓度与疾病的发病率和致死率高度相关 (Hanna et al., 2011; Vanos et al., 2015)。因此，研究大气污染物对 COVID-19 传播的影响，为各地区的 干预政策制定者提供一个方向 (Anderson et al., 2009)。

此外，根据 2020 年 3 月 3 日国家卫生健康委员会发布的《新型冠状病毒肺炎诊疗方案 (试行第七版) 》中华人民共和国国家卫生健康委员会 (2020), 经呼吸道飞沫和密切接触传播是 COVID-19 主要的传播途径, 在相对封闭的环境中长期暴露于高浓度气溶胶情况下存在气溶胶 传播可能。气溶胶传播即意味着 SARS-CoV-2 可能可以在空气中存活一段时间，因此，探究影 响空气流通的日平均温度、日平均相对湿度、日平均风速和空气污染物等因素便变得更加重要。

本文以人口密集、非输入型病例发病率较高的 2 个城市，即深圳市和温州市作为研究对象, 通过对两个城市每日的新增非输入型确诊人数与前期气象数据的对比分析, 比较不同气象因素与 空气质量对各城市的影响，探索 COVID-19 的感染与特定气象条件的关系, 以期为该病预防和 机理研究提供新的科学依据。

\section{2 资料与方法}

\section{1 数据来源}

\subsection{1 气象数据}

为了探索每天的温度 $\left({ }^{\circ} \mathrm{C}\right)$ 、湿度 $(\%)$ 、和风速 $(\mathrm{km} / \mathrm{h})$ 等是否可能影响 SARS-CoV-2 在空气 中的传播, 我们收集了温度、湿度和风速数据, 数据来源于美国公司 Weather Underground(The Weather Company, an IBM business, 2020)，该公司提供世界范围内各城市的气象数据，3 小时 更新一次。由于我们进行分析的数据以天为单位, 故我们对每天的温度、湿度和风速数据做平均 值处理，用以代替当天的气象情况。本文收集了自 2020 年 1 月 1 日到 2020 年 2 月 29 日共 60 天的温度、湿度及风速数据以供分析，以下简称气象因素。折线图趋势表见附录A。

\subsection{2 空气质量数据}

根据 “真气网” 中的数据以及国家发布的《环境空气质量指数技术规定（试行)》(王艳琴, $2012)$, 大气污染物通常指二氧化氮 $\left(\mathrm{NO}_{2}: \mu \mathrm{g} / \mathrm{m}^{3}\right)$ 、粒径小于等于 2.5 微米的颗粒物 $\left(\mathrm{PM}_{2.5}\right.$ : 


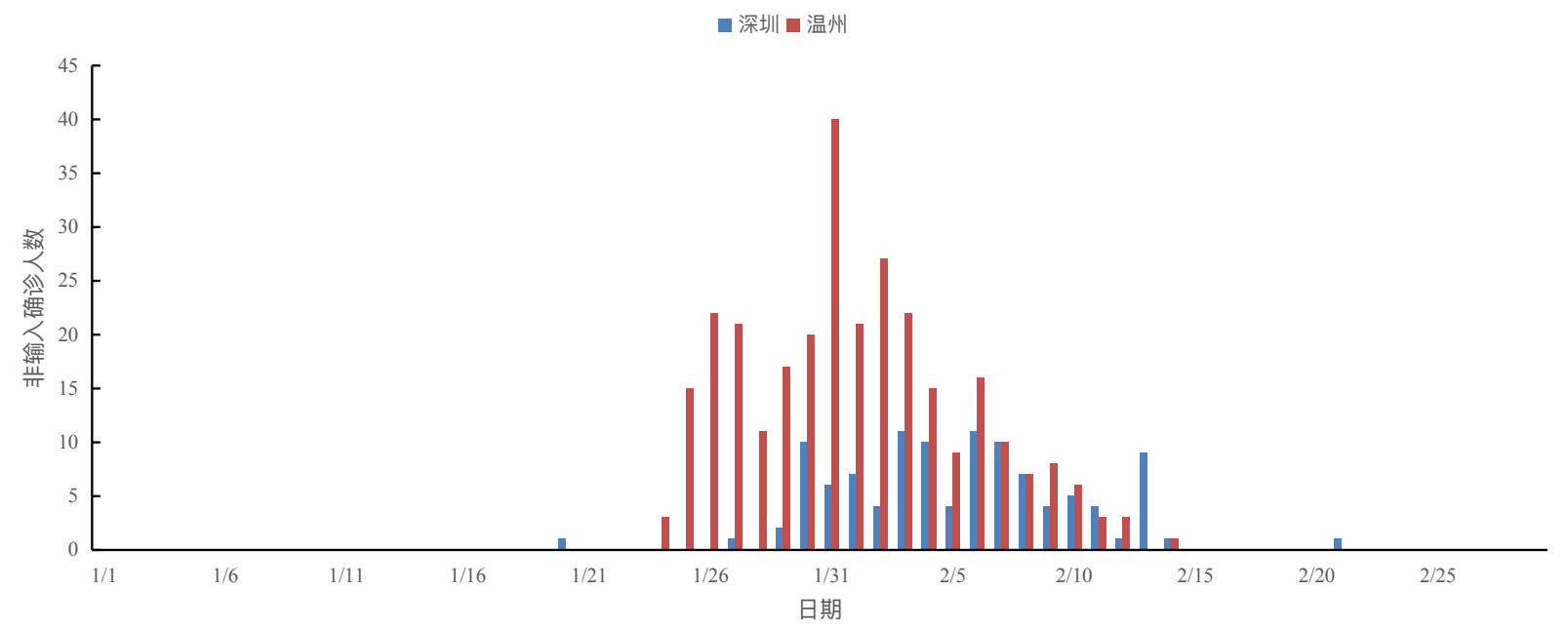

图 1: 非输入确诊人数柱状图。

$\left.\mu \mathrm{g} / \mathrm{m}^{3}\right)$ 、粒径小于等于 10 微米的颗粒物 $\left(\mathrm{PM}_{10}: \mu \mathrm{g} / \mathrm{m}^{3}\right)$ 、一氧化碳 $\left(\mathrm{CO}: \mathrm{mg} / \mathrm{m}^{3}\right)$ 、臭氧 $\left(\mathrm{O}_{3}: \mu \mathrm{g} / \mathrm{m}^{3}\right)$ 和二氧化硫 $\left(\mathrm{SO}_{2}: \mu \mathrm{g} / \mathrm{m}^{3}\right)$ 等。由于 $\mathrm{SO}_{2}$ 数据过于平稳, 对结果影响较小, 故排 除 $\mathrm{SO}_{2}$ 指标; 同时, 由于 $\mathrm{PM}_{2.5}$ 和 $\mathrm{PM}_{10}$ 具有较强的共线性 (见附录 $\mathrm{A}$. 折线图), 因此我们只 保留 $\mathrm{PM}_{2.5}$ 进行后续分析，最终将 $\mathrm{NO}_{2} 、 \mathrm{PM}_{2.5} 、 \mathrm{CO}$ 和 $\mathrm{O}_{3}$ （日最大 8 小时平均） 4 项检测的 污染物作为本文中的空气质量指标。文章收集了自 2020 年 1 月 1 日到 2020 年 2 月 29 日共 60 天的上述指标以供分析，以下简称大气污染因素，折线图趋势表见附录A.。

\subsubsection{COVID-19 确诊数据}

每日确诊病例数据来源于深圳市、温州市两地的卫生健康委员会。病例数据包括各地确诊病 例、确诊非输入型病例、各病例发病日期（定义为病例自我报告第一次开始发烧或咳嗽的日期）、 每日累计治愈病例以及每日累计死亡病例。湖北省作为中国 COVID-19 疫情最严重的地区，我 们将输入型病例的定义为: 有湖北旅居史的病例, 非输入型为无湖北旅居史的病例。输入型病例 主要受到湖北迁出至该城市的人口多少的影响, 但非输入型病例的人数可能与人与人接触密度、 空气传播等有关系。因此本文考虑每日新增的非输入型确诊病例，以下简称确诊人数。根据文 献Li et al. (2020), SARS-CoV-2 的潜伏期可为 14 天, 由于气象和空气质量的数据从 2020 年 1 月 1 日起收集，我们考虑最长滞后 14 天的效应，因此，从 2020 年 1 月 15 日开始收集深圳、温 州两个城市的确诊人数，至 2020 年 2 月 29 日，共 45 天数据。

图1为深圳、温州市每日新增的非输入型确诊人数的柱状图, 其中蓝色柱体表示深圳人数、红 色柱体表示温州人数。由图1可知, 从 1 月 20 日起, 深圳市开始出现非输入型确诊病例, 而温州 则从 1 月 24 日开始出现。深圳、温州市的疫情情况均在 2 月 20 日前趋于稳定，2 月 20 日后， 2 座城市的非输入型确诊人数均保持为 0 。从图上可知, 深圳有确诊人数的天数为 20 天，温州 有 21 天。因此, 确诊人数为 0 的天数在我们的数据收集时间内，深圳占比为 $55.6 \%$, 温州占比 为 $53.3 \%$ 。 


\section{2 研究方法}

本文中所有的数据整理、统计分析均使用 3.6 版本的 R 软件进行处理 (路凤等, 2018), 文章 代码可参见：https://github.com/CurlySheep/Air-COVID。

\subsection{1 分布滞后非线性模型}

一般流行病学、常见的混杂因素包括年龄、性别、体重指数、吸烟状况、喝酒等等，但这些 “标准混杂因素” 并不适用于我们的数据, 因为在人口层面, 这些因素的分布没有 (或不可能) 逐 日改变, 并且不能影响污染程度等环境因素的波动。因此, 潜在的混杂因素应该是每天都在变化 的变量, 这些变量与我们感兴趣的暴露的每日波动（气象因素和大气污染因素）以及结果（确诊 人数）有关 (Gasparrini et al., 2015)。

考虑到气象与空气污染等因素对疾病的影响往往具有非线性的滞后效应，以广义线性模型 为基础，本文使用分布滞后非线性模型（Distributed Lag Non-linear Model, DLNM）来评估各 因素对 SARS-CoV-2 传播的影响。

首先考虑基础模型Gasparrini (2011); Gasparrini et al. (2010, 2013); 杨军等 (2012) :

$$
g\left(\mu_{t}\right)=\alpha+\sum_{j=1}^{J} s_{j}\left(X_{t j} ; \boldsymbol{\beta}_{j}\right)
$$

其中 $g$ 为连接函数，文章中选为 $g(x)=\ln (x)$ 。 $\mu_{t}=E\left(Y_{t}\right), Y_{t}$ 为第 $t$ 天的因变量, $s_{j}$ 为变量 $X_{t j}$ 的基函数, $\boldsymbol{\beta}_{j}$ 为对应方程中的参数向量, $t=1, \cdots, T, T$ 为总天数， $J$ 为变量个数。

引入基函数后，我们将原始变量转化为一系列新的基变量，通过转化可更好的描述因变量随 自变量变化的分布。下面考虑基函数 $s_{j}$ （这里我们的文章中分别选取交叉基函数 $C B$, 与自然三 次 B 样条函数 $N S$ ):

$$
s_{j}\left(X_{t} ; \boldsymbol{\beta}_{j}\right)=\mathbf{z}_{t}^{\mathrm{T}} \boldsymbol{\beta}_{j},
$$

$\mathbf{z}_{t}=\left[z_{t 1}, \cdots, z_{t v_{x}}\right]^{\top}$ 是 $T \times v_{x}$ 矩阵 $\mathbf{Z}$ 的第 $t$ 行的元素, $v_{x}$ 为转化的新变量的维度。

为了考虑变量的滞后性，我们对选定的一个自变量 $X_{t j}$, 考虑其在时间维度 $0, \cdots, L$ 上的变 化, $L$ 为最长滞后天数, 而后根据时间维度产生序列 $q_{t} .=\left[X_{t j}, \cdots, X_{t-L, j}\right]$, 反映该因素的滞后 效应，记为 $T \times(L+1)$ 的矩阵 $\mathbf{Q}$ 。由此引入分布滞后模型（Distributed Lag Models, DLMs)：

$$
s_{j}\left(x_{t} ; \boldsymbol{\eta}_{j}\right)=\sum_{k=1}^{v_{\ell}} \mathbf{q}_{t}^{\top} \cdot \mathbf{c}_{\cdot k} \eta_{k j}=\mathbf{q}_{t}^{\top} \mathbf{C} \boldsymbol{\eta}_{j},
$$

其中 $\mathbf{c}_{. k}$ 为 $(L+1) \times v_{\ell}$ 的矩阵 $\mathbf{C}$ 的第 $k$ 列，其为对滞后向量选择特定的基函数转换所得到， $\boldsymbol{\eta}_{j}$ 为每个滞后时间的线性效应。

而后我们引入交叉基（cross-basis）函数，其思想考虑选择的因素与因变量的非线性关系及 对应的效应滞后性，分别选择合适的基函数，求两个基函数的张量积即可得到交叉基函数（文 章中考虑的是自然三次样条二维函数)，其能够提高模型的适用性和结果的可靠性 (Gasparrini et al., 2010)。因此将前面(1)与(2)两式结合，既考虑因变量随自变量变化所选取的基函数，又考 
虑滞后效应，DLNM 的交叉基函数 $s_{j}\left(x_{t} ; \boldsymbol{\eta}_{j}\right)$ 可写为:

$$
s_{j}\left(x_{t} ; \boldsymbol{\eta}_{j}\right)=\sum_{i=1}^{v_{x}} \sum_{k=1}^{v_{\ell}} \mathbf{r}_{t i}^{\top} \cdot \mathbf{c}_{\cdot} \eta_{i k j}=\mathbf{w}_{t}^{\top} \boldsymbol{\eta}_{j}
$$

这里, $\mathbf{r}_{t i}=\left[r_{t i 1}, \cdots, r_{t i,(L+1)}\right]^{\top}$ 是 $T \times v_{x} \times(L+1)$ 的三维序列 $\mathbf{R}$ 中的向量, $\mathbf{w}_{t}$ 为交叉基矩 阵 $\mathbf{W}$ 的第 $t$ 行。

我们以确诊人数作为因变量, 气象因素和大气污染因素作为自变量, 在广义线性模型的基 础上, 建立多因素分布滞后模型分别定量评估气象因素和大气污染因素对确诊人数的滞后相关 性。由于 COVID-19 的发病为小概率事件且存在过度离散化的现象, 我们使用对数函数作为模 型的连接函数。其中, 将各个主要研究的气象因素和大气污染因素以交叉基的形式纳入, 其余变 量我们使用自然三次 B 样条函数进行非参数光滑处理 (段振华等, 2015; McMichael et al., 2008; Gasparrini et al., 2013; Knott, 2000), 并根据 COVID-19 传播的潜伏期,将最大滞后天数设定为 14 天以观察气象因素和大气污染因素对 COVID-19 传染影响的滞后分布特点 (Armstrong, 2006)。 针对不同指标拟合传染病的日病例数离散程度不一的问题，本文采用拟泊松（quasi-Poisson）分 布建立模型。相比于一般的泊松回归, 拟泊松回归引入了过离散参数 $\phi$ (dispersion parameter), 即方差与期望的关系用此参数进行联系，而不是默认方差与期望相等。具体表达如下式所示：

$$
E\left(Y_{t}\right)=\mu_{t}, \quad \operatorname{Var}\left(Y_{t}\right)=\phi \mu_{t}
$$

连接函数我们选择 $\log (\cdot)$ 。因此，利用拟泊松分布建立模型的表达式如下:

$$
\log \left[E\left(Y_{t}\right)\right]=\alpha+C B\left(X_{t i}, l a g\right)+\sum_{j \neq i} N S\left(X_{t j}, d f\right)
$$

上式中: $Y_{t}$ 为第 $t$ 日的确诊非输入病例数, $E\left(Y_{t}\right)$ 为第 $t$ 日的确诊非输入病例数的期望值; $X_{t i}$ 为希望研究的第 $t$ 日的某一个气象因素或大气污染因素, $l a g$ 为最长滞后天数, $C B\left(X_{t}, l a g\right)$ 则 表示对 $X_{t}$ 构建的最长滞后天数为 $l a g$ 的交叉基，是通过 DLNM 作用于 $X_{t}$ 而获得的矩阵; $N S$ 为自然三次 B 样条函数, $X_{j t}$ 为除 $X_{i t}$ 外剩余的所有第 $\mathrm{t}$ 日气象因素及大气污染因素, $d f$ 表示自 由度, $N S\left(X_{j t}, d f\right)$ 表示具有自由度为 $d f$ 的 $X_{j t}$ 的自然三次样条函数, 用以消除长期影响。模型 中自由度的确定参考文献的设置 (Gasparrini et al., 2010; Runkle et al., 2020; 谷少华等, 2016), 我们选为 3 。

为更好的解释模型的构建过程，我们以某一天的温度为例，构建的模型如下所示：

$$
\begin{aligned}
\log [E(Y)]= & \alpha+C B(\text { temperature }, \text { lag })+N S(\text { humidity }, d f)+N S(\text { windspeed }, d f) \\
& +N S\left(P M_{2.5}, d f\right)+N S(C O, d f)+N S\left(O_{3}, d f\right) .
\end{aligned}
$$

\subsection{2 相对危险度 (Relative Risk, RR)}

由于 DLNM 对每一个暴露变量均估计了大量的回归系数, 暴露变量-响应变量的相关性难以 直观地用估计系数进行总结, 故引入了相对危险度 (RR) 来衡量气象因素和大气污染因素对确诊 
人数的滞后影响 (杨军等, 2012)。排除长期趋势后，考虑气象因素和大气污染因素对 COVID-19 发病可能存在滞后效应, 将 0（即当天）-14 天前的气象因素和大气污染因素分别引入模型，估 算回归系数 $\boldsymbol{\beta}$ ，计算 RR 和 $95 \%$ 可信区间（95\% Confidence Interval：95\% CI），基准水平为各 气象因素和大气污染因素的中位数 (宋捷, 2015)。相对危险度及其置信区间的计算公式为：

$$
\begin{aligned}
R R & =\exp (\boldsymbol{\beta} \mathbf{x}), \\
R R \text { 的 } 95 \% C I \text { 上下界 } & =\exp [(\boldsymbol{\beta} \pm 1.96 \cdot S E) \cdot \mathbf{x}],
\end{aligned}
$$

其中, $S E$ 为回归系数估计的标准差, $\mathrm{x}$ 为文章中考虑到的气象因素或大气污染因素的变化 量向量, 其中每一个元素 $x$ 为具体的某一因素。以温度为例, 设置 $10^{\circ} \mathrm{C}$ 为基准, 当 $x$ 的变化为 $5^{\circ} \mathrm{C}$ 时，则将 5 代入上式中，即为求取 $15^{\circ} \mathrm{C}$ 的 $\mathrm{RR}$ 值，这表示当气温从 $10^{\circ} \mathrm{C}$ 上升到 $15^{\circ} \mathrm{C}$ 时， 气温对疫情传播造成的影响。反之, 将 -5 代入上式中, 即为求取 $5^{\circ} \mathrm{C}$ 的 $\mathrm{RR}$ 值, 这表示当气温从 $10^{\circ} \mathrm{C}$ 下降到 $5^{\circ} \mathrm{C}$ 时，气温对疫情传播造成的影响。若 RR $>1$, 则表示对 COVID-19 疫情传播 的影响为正，气温升高或降低可加剧 COVID-19 疫情的传播; 若 $R R<1$, 则表示对 COVID-19 疫情的传播起到抑制作用。

\subsection{3 两城市对比分析}

我们将对比评估深圳市和温州市中的非输入型累计确诊人数受其所在城市的气象因素和大 气污染因素的影响。对每个气象因素指标（日平均温度、日平均相对湿度和日平均风速）和空气 质量指标 $\left(\mathrm{NO}_{2} 、 \mathrm{PM}_{2.5} 、 \mathrm{CO}\right.$ 和 $\left.\mathrm{O}_{3}\right)$ 中每一个指标的范围在两个城市中合并, 即两个城市中的 任意一个指标的最小的取值作为这个指标的最小值，而该指标的两个城市中的最大的取值作为 指标的最大值。也就是说，两个城市通过数据合并后，每个指标都将包含两个城市该指标的所有 数据范围。在每个指标的取值范围确定后，将其所对应的中位数作为该指标的基准水平。用每个 指标中的数值范围排序在 $[10 \%, 90 \%]$ 之间，每间隔 $10 \%$ 的数据计算其与基准水平相比较的 RR 及对应的 $95 \% \mathrm{CI}$ 的可信区间。

\section{3 结果}

本文对深圳市和温州市的日平均温度、日平均相对湿度、日平均风速、 $\mathrm{NO}_{2} 、 \mathrm{PM}_{2.5} 、 \mathrm{CO}$ 和 $\mathrm{O}_{3}$ 共 7 项指标进行 DLNM 拟合。分别计算各指标 0-14 天滞后天数的 RR 值，依据 RR 值及 其对应的置信区间判断该指标是否对非输入确诊病例人数具有影响。模型的离散参数估计结果 如附录B.所示; 模型的拟合情况如附录C.所示; 深圳市、温州市的气象与大气污染因素与非输入 确诊风险关联情况表如附录D.所示。

\section{1 气象因素}

\subsection{1 日平均温度}

将每日平均温度作为首要研究指标进行考虑。模型拟合结果显示, 非输入确诊人数的 RR 与 每日平均温度存在复杂的非线性关系。表 1 为深圳市、温州市的每日平均温度与基线日平均温度 
表 1: 深圳、温州市平均温度与非输入确诊风险关联情况。

\begin{tabular}{|c|c|c|c|c|}
\hline \multirow{3}{*}{ 滞后天数 } & \multicolumn{2}{|c|}{$11^{\circ} \mathbf{C}(\mathbf{3 0 \%})$} & \multicolumn{2}{|c|}{$18^{\circ} \mathbf{C}(\mathbf{7 0 \%})$} \\
\hline & 深圳 & 温州 & 深圳 & 温州 \\
\hline & $\mathrm{RR}(95 \% \mathrm{CI})$ & $\mathrm{RR}(95 \% \mathrm{CI})$ & $\mathrm{RR}(95 \% \mathrm{CI})$ & $\mathrm{RR}(95 \% \mathrm{CI})$ \\
\hline 0 & $0.33(0.07,1.70)$ & $0.82(0.56,1.21)$ & $0.73(0.42,1.27)$ & $7.03(2.72,18.15)^{*}$ \\
\hline 1 & $0.42(0.12,1.47)$ & $1.05(0.82,1.35)$ & $0.80(0.54,1.18)$ & $5.43(2.14,13.74)^{*}$ \\
\hline 2 & $0.54(0.21,1.37)$ & $1.31(1.07,1.62)^{*}$ & $0.87(0.67,1.13)$ & $4.16(1.28,13.51)^{*}$ \\
\hline 3 & $0.67(0.32,1.40)$ & $1.59(1.23,2.06)^{*}$ & $0.93(0.77,1.13)$ & $3.16(0.74,13.42)$ \\
\hline 4 & $0.80(0.41,1.58)$ & $1.86(1.35,2.56)^{*}$ & $0.98(0.81,1.18)$ & $2.36(0.46,12.04)$ \\
\hline 5 & $0.94(0.48,1.86)$ & $2.10(1.46,3.00)^{*}$ & $1.01(0.82,1.25)$ & $1.75(0.32,9.59)$ \\
\hline 6 & $1.08(0.53,2.18)$ & $2.29(1.58,3.33)^{*}$ & $1.03(0.82,1.30)$ & $1.28(0.24,6.81)$ \\
\hline 7 & $1.20(0.58,2.50)$ & $2.44(1.70,3.51)^{*}$ & $1.04(0.81,1.33)$ & $0.93(0.20,4.38)$ \\
\hline 8 & $1.32(0.62,2.81)$ & $2.54(1.81,3.55)^{*}$ & $1.03(0.79,1.34)$ & $0.67(0.17,2.59)$ \\
\hline 9 & $1.42(0.65,3.13)$ & $2.58(1.91,3.50)^{*}$ & $1.02(0.77,1.34)$ & $0.48(0.16,1.46)$ \\
\hline 10 & $1.52(0.65,3.53)$ & $2.59(1.95,3.43)^{*}$ & $1.00(0.74,1.34)$ & $0.34(0.14,0.82)^{*}$ \\
\hline 11 & $1.60(0.63,4.07)$ & $2.55(1.90,3.43)^{*}$ & $0.97(0.70,1.35)$ & $0.24(0.11,0.52)^{*}$ \\
\hline 12 & $1.67(0.58,4.84)$ & $2.49(1.76,3.53)^{*}$ & $0.94(0.64,1.38)$ & $0.17(0.07,0.42)^{*}$ \\
\hline 13 & $1.74(0.51,5.91)$ & $2.42(1.57,3.74)^{*}$ & $0.91(0.58,1.42)$ & $0.12(0.03,0.41)^{*}$ \\
\hline 14 & $1.80(0.44,7.37)$ & $2.34(1.36,4.03)^{*}$ & $0.88(0.52,1.48)$ & $0.08(0.02,0.45)^{*}$ \\
\hline
\end{tabular}

相比后的详细 $\mathrm{RR}$ 值及其置信区间。其中，以 $15^{\circ}$ 为基准日平均温度，表 1 中各 RR 值表示不同 日平均温度比基准日平均温度 $\left(15^{\circ} \mathrm{C}\right)$ 低或高时的 COVID-19 的相关危险度，* 表示该滞后天 数下 RR 值及 $R R$ 的置信区间均处于 1 的同一侧，即该结果显著。

由表 1 可知，当日平均温度为 $11^{\circ} \mathrm{C}$ 时，与日平均温度为 $15^{\circ} \mathrm{C}$ 时相比，日平均温度的降低， 加剧了温州市的 COVID-19 的疫情传播风险。具体来说; 当滞后天数为 2-14 天时，该日平均温 度加剧了温州市的非输入确诊病例增加的风险（RR>1，95\%CI $=(1,3.6))$ 。

当日平均温度为 $18^{\circ} \mathrm{C}$ 时, 当滞后天数为 $0-2$ 天时，该日平均温度加剧了温州市的非输入确 诊病例增加的风险（ $\mathrm{RR}>1 ， 95 \% \mathrm{CI}=(1,18.2))$; 同温度下，当滞后天数为 $10-14$ 天时，该日 平均温度降低了温州市非输入确诊病例的风险 $(\mathrm{RR}<1,95 \% \mathrm{CI}=(0,1))$ 。

\subsection{2 日平均相对湿度}

将日平均相对湿度作为首要研究指标进行考虑。模型拟合结果显示, 非输入确诊人数的 RR 与相对湿度存在复杂的非线性关系。表 2 为深圳市、温州市的不同日平均相对湿度与非输入型确 诊病例的详细 RR 值及其置信区间。其中，以 $76 \%$ 为基准，表 2 中各 RR 值表示不同日平均相 对湿度比 $76 \%$ 低或高时的 COVID-19 的相关危险度, * 表示该滞后天数下 RR 值及 RR 的置信 区间均处于 1 的同一侧，表示该处的结果较为显著。

由表 2 可知，当日平均相对湿度的百分比为 $69.25 \%$ 时，相对湿度的降低对深圳市疫情无显 著影响。当滞后天数为 9-14 天时, 日平均相对湿度的降低使温州市非输入型确诊人数增加的风 险降低（RR<1，95\% CI= $(0,1) ＼mathrm{~ 。 ~}$

当日平均相对湿度上升为 $81 \%$ 时，滞后天数为 12-13 天时，该相对湿度增加了深圳市非输 
表 2: 深圳、温州市日平均相对湿度与非输入确诊风险关联情况。

\begin{tabular}{|c|c|c|c|c|}
\hline \multirow{3}{*}{ 滞后天数 } & \multicolumn{2}{|c|}{$69 \%(\mathbf{3 0 \%})$} & \multicolumn{2}{|c|}{$81 \%(\mathbf{7 0 \%})$} \\
\hline & 深圳 & 温州 & 深圳 & 温州 \\
\hline & $\mathrm{RR}(95 \% \mathrm{CI})$ & $\mathrm{RR}(95 \% \mathrm{CI})$ & $\mathrm{RR}(95 \% \mathrm{CI})$ & $\mathrm{RR}(95 \% \mathrm{CI})$ \\
\hline 0 & $1.36(0.75,2.47)$ & $1.48(0.56,3.90)$ & $0.80(0.54,1.19)$ & $0.63(0.38,1.03)$ \\
\hline 1 & $0.94(0.50,1.78)$ & $1.31(0.44,3.93)$ & $0.96(0.65,1.40)$ & $0.59(0.32,1.07)$ \\
\hline 2 & $0.68(0.31,1.49)$ & $1.14(0.33,3.91)$ & $1.13(0.72,1.77)$ & $0.57(0.28,1.15)$ \\
\hline 3 & $0.53(0.21,1.34)$ & $0.97(0.25,3.70)$ & $1.30(0.76,2.24)$ & $0.57(0.26,1.27)$ \\
\hline 4 & $0.44(0.16,1.26)$ & $0.80(0.19,3.30)$ & $1.45(0.78,2.70)$ & $0.61(0.26,1.43)$ \\
\hline 5 & $0.40(0.13,1.21)$ & $0.64(0.15,2.79)$ & $1.58(0.81,3.10)$ & $0.68(0.28,1.65)$ \\
\hline 6 & $0.39(0.13,1.17)$ & $0.50(0.11,2.26)$ & $1.68(0.83,3.38)$ & $0.79(0.32,1.94)$ \\
\hline 7 & $0.40(0.14,1.15)$ & $0.38(0.08,1.76)$ & $1.74(0.86,3.52)$ & $0.96(0.40,2.33)$ \\
\hline 8 & $0.43(0.16,1.15)$ & $0.29(0.06,1.33)$ & $1.77(0.89,3.52)$ & $1.21(0.51,2.88)$ \\
\hline 9 & $0.48(0.20,1.16)$ & $0.21(0.05,0.99)^{*}$ & $1.78(0.93,3.41)$ & $1.56(0.66,3.67)$ \\
\hline 10 & $0.56(0.26,1.19)$ & $0.16(0.03,0.74)^{*}$ & $1.76(0.96,3.22)$ & $2.07(0.88,4.86)$ \\
\hline 11 & $0.67(0.35,1.26)$ & $0.11(0.02,0.55)^{*}$ & $1.72(0.99,3.00)$ & $2.79(1.16,6.72)^{*}$ \\
\hline 12 & $0.82(0.47,1.42)$ & $0.08(0.02,0.42)^{*}$ & $1.67(1.00,2.78)^{*}$ & $3.83(1.51,9.69)^{*}$ \\
\hline 13 & $1.01(0.59,1.75)$ & $0.06(0.01,0.32)^{*}$ & $1.61(1.00,2.60)^{*}$ & $5.29(1.93,14.52)^{*}$ \\
\hline 14 & $1.27(0.68,2.39)$ & $0.04(0.01,0.25)^{*}$ & $1.55(0.97,2.47)$ & $7.36(2.42,22.42)^{*}$ \\
\hline
\end{tabular}

入确诊病例增加的风险 $(\mathrm{RR}>1,95 \% \mathrm{CI}=(1,2.8))$; 同样相对湿度下, 滞后天数为 11-14 天时, 该相对湿度也增加了温州市非输入确诊病例增加的风险（RR>1，95\% CI $=(1,22.5)$ 。

\subsection{3 日平均风速}

将日平均风速作为首要研究指标进行考虑。模型拟合结果显示, 深圳市日平均风速则对深圳 市疫情有较弱的影响, 同样, 温州市的非输入确诊人数的 RR 与日平均风速存在较弱的非线性 关系。表3 为深圳市、温州市不同日平均风速与非输入型确诊病例的详细 RR 值及其置信区间。 其中, 以 $7.00 \mathrm{~km} / \mathrm{h}$ 为基准日平均风速，表 3 中各 $\mathrm{RR}$ 值表示不同日平均风速比基准日平均风速 $(7.00 \mathrm{~km} / \mathrm{h})$ 低或高时的 COVID-19 相关危险度，* 表示该滞后天数下 RR 值及 RR 的置信区 间均处于 1 的同一侧，表示该处的结果较为显著。

由表 3 可知, 对于深圳市, 日平均风速对疫情的影响较弱, 仅在日平均风速为 $5.75 \mathrm{~km} / \mathrm{h}$ 且 滞后天数为 0 天时，日平均风速的降低增加了深圳市的疫情风险（RR> $1 ， 95 \% \mathrm{CI}=(1,4.4)$ 。 同样日平均风速下，滞后天数分别为 2-9 天时，日平均风速的降低使温州市的 COVID-19 疫情 得到缓解 $(\mathrm{RR}<1,95 \% \mathrm{CI}=(0,1))$ 。

为进一步探究日平均风速对非输入确诊的影响，现增加日平均风速下降和升高的范围，即 日平均风速下降为 $4.86 \mathrm{~km} / \mathrm{h}(10 \%)$ 和 $10.00 \mathrm{~km} / \mathrm{h}(90 \%)$ 时的 RR 与其置信区间的二维图, 如 图2。其中, 红色实心线表示深圳市日平均风速在对应数值处的 RR 值, 红色区域表示其 $95 \%$ 置 信区间; 蓝色实心线表示温州市日平均风速在对应数值处的 RR 值, 蓝色区域表示其 $95 \%$ 置信 区间。左侧二维图表示日平均风速为 $4.86 \mathrm{~km} / \mathrm{h}(10 \%)$ 时的 RR 情况，右侧二维图表示日平均 
表 3: 深圳、温州市日平均风速与非输入确诊风险关联情况。

\begin{tabular}{|c|c|c|c|c|}
\hline \multirow{3}{*}{ 滞后天数 } & \multicolumn{2}{|c|}{$5.75 \mathrm{~km} / \mathrm{h}(\mathbf{3 0 \%})$} & \multicolumn{2}{|c|}{$8.00 \mathrm{~km} / \mathrm{h}(\mathbf{7 0 \%})$} \\
\hline & 深圳 & 温州 & 深圳 & 温州 \\
\hline & $\mathrm{RR}(95 \% \mathrm{CI})$ & $\mathrm{RR}(95 \% \mathrm{CI})$ & $\mathrm{RR}(95 \% \mathrm{CI})$ & $\mathrm{RR}(95 \% \mathrm{CI})$ \\
\hline 0 & $2.07(1.00,4.30)^{*}$ & $1.18(0.76,1.82)$ & $1.07(0.64,1.79)$ & $1.25(0.91,1.70)$ \\
\hline 1 & $1.67(0.89,3.12)$ & $0.56(0.29,1.09)$ & $0.89(0.56,1.39)$ & $1.35(0.96,1.90)$ \\
\hline 2 & $1.37(0.77,2.44)$ & $0.29(0.10,0.89)^{*}$ & $0.75(0.40,1.38)$ & $1.45(0.94,2.22)$ \\
\hline 3 & $1.17(0.66,2.07)$ & $0.18(0.04,0.80)^{*}$ & $0.65(0.29,1.47)$ & $1.52(0.91,2.54)$ \\
\hline 4 & $1.05(0.58,1.89)$ & $0.13(0.02,0.75)^{*}$ & $0.59(0.22,1.57)$ & $1.55(0.87,2.77)$ \\
\hline 5 & $0.98(0.53,1.80)$ & $0.11(0.02,0.73)^{*}$ & $0.55(0.18,1.64)$ & $1.56(0.84,2.87)$ \\
\hline 6 & $0.94(0.50,1.77)$ & $0.10(0.01,0.74)^{*}$ & $0.53(0.17,1.68)$ & $1.53(0.82,2.84)$ \\
\hline 7 & $0.94(0.50,1.80)$ & $0.11(0.02,0.77)^{*}$ & $0.52(0.16,1.68)$ & $1.48(0.81,2.70)$ \\
\hline 8 & $0.97(0.50,1.88)$ & $0.13(0.02,0.83)^{*}$ & $0.53(0.17,1.64)$ & $1.41(0.80,2.48)$ \\
\hline 9 & $1.02(0.51,2.05)$ & $0.17(0.03,0.92)^{*}$ & $0.54(0.19,1.59)$ & $1.33(0.80,2.20)$ \\
\hline 10 & $1.10(0.52,2.31)$ & $0.25(0.06,1.04)$ & $0.57(0.22,1.51)$ & $1.23(0.80,1.90)$ \\
\hline 11 & $1.20(0.54,2.70)$ & $0.37(0.11,1.22)$ & $0.61(0.26,1.43)$ & $1.14(0.80,1.62)$ \\
\hline 12 & $1.33(0.54,3.26)$ & $0.59(0.23,1.51)$ & $0.65(0.31,1.36)$ & $1.04(0.80,1.37)$ \\
\hline 13 & $1.49(0.55,4.05)$ & $0.97(0.46,2.02)$ & $0.70(0.38,1.30)$ & $0.95(0.78,1.17)$ \\
\hline 14 & $1.66(0.54,5.11)$ & $1.60(0.80,3.20)$ & $0.76(0.45,1.29)$ & $0.87(0.72,1.05)$ \\
\hline
\end{tabular}

风速为 $10.00 \mathrm{~km} / \mathrm{h}(90 \%)$ 时的 $\mathrm{RR}$ 情况。

由图 2 可知, 当日平均风速为 $4.86 \mathrm{~km} / \mathrm{h}(10 \%)$, 且滞后天数为 6-8 天时, 降低深圳市日平均 风速, 可使深圳市非输入确诊人数增加的风险降低 $(\mathrm{RR}<1,95 \% \mathrm{CI}=(0,1))$; 同样日平均风速 下，当滞后天数为 2-10 天时，温州市的 COVID-19 的相对危险降低（RR<1，95\%CI = $(0,1)$ 。 当日平均风速为 $10.00 \mathrm{~km} / \mathrm{h}(90 \%)$, 且滞后天数为 $2-11$ 天时, 日平均风速的增加缓解了温 州市非输入确诊人数增加的风险 $(\mathrm{RR}<1,95 \% \mathrm{CI}=(0,1))$ 。

总的来说，以 $7.00 \mathrm{~km} / \mathrm{h}$ 为基准时，不论是降低日平均风速或提高日平均风速，疫情传播 的风险均会随之降低。即在日平均风速为 $7.00 \mathrm{~km} / \mathrm{h}$ 左右时, 可能为 COVID-19 疫情传播的最 适合日平均风速，即此时疫情传播的风险最大。

\section{2 空气污染因素}

\subsection{1 $\quad \mathrm{NO}_{2}$}

$\mathrm{NO}_{2}$ 作为首要研究指标放入 DLNM 模型后, 拟合结果显示, 非输入确诊人数的 $\mathrm{RR}$ 与 $\mathrm{NO}_{2}$ 在深圳、温州市之间，存在相反的非线性关系。表 4 为深圳市与温州市 $\mathrm{NO}_{2}$ 与非输入型确诊病例 的详细 $\mathrm{RR}$ 值及其置信区间。其中， $\mathrm{RR}$ 值以 $16 \mu \mathrm{g} / \mathrm{m}^{3}$ 为基准，表 4 中各 $\mathrm{RR}$ 值表示不同浓度 与 $16 \mu \mathrm{g} / \mathrm{m}^{3}$ 相比时 COVID-19 疫情的相关危险度, * 表示该滞后天数下 RR 值及 RR 的置信 区间均处于 1 的同一侧，表示该处的结果较为显著。

由表4可知, 当 $\mathrm{NO}_{2}$ 的浓度为 $11 \mu \mathrm{g} / \mathrm{m}^{3}$ 且滞后天数为 1-4 天时, $\mathrm{NO}_{2}$ 浓度的降低将使深圳 市的非输入确诊感染风险上升 $(\mathrm{RR}>1,95 \% \mathrm{CI}=(1,27.34))$; 同样浓度下，当滞后天数为 8-14 


\section{city $=$ 深圳 $\multimap$ 温州}

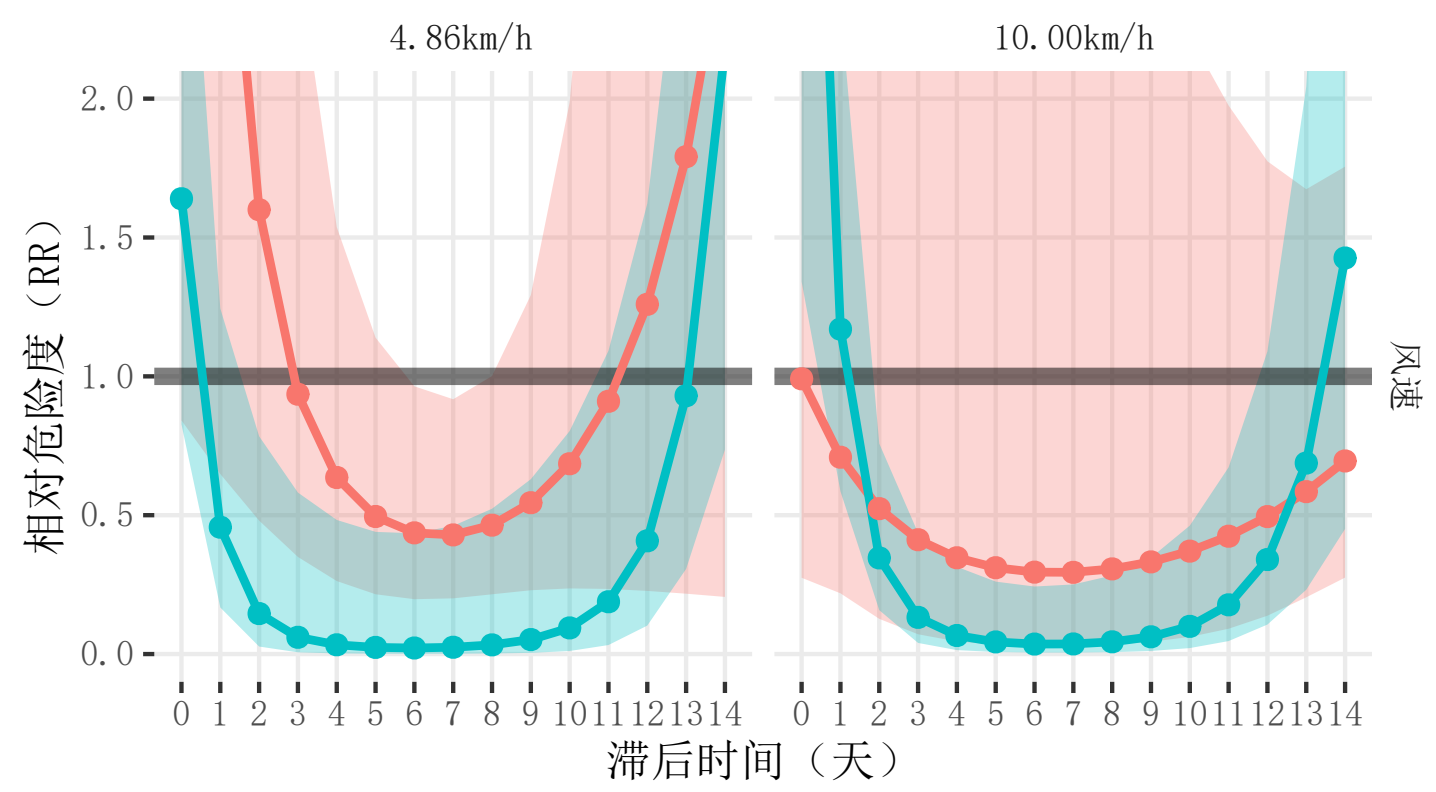

图 2: 不同日平均风速（左： $4.86 \mathrm{~km} / \mathrm{h}(10 \%)$; 右： $10 \mathrm{~km} / \mathrm{h}(10 \%)$ ）与基准日平均风速比较后, 滞后 0-14 天的 RR 二维图。

天时， $\mathrm{NO}_{2}$ 浓度的降低将使温州市的非输入确诊感染风险下降（RR $<1 ， 95 \% \mathrm{CI}=(0,1)$ 。

\subsection{2 $\quad \mathrm{PM}_{2.5}$}

将 $\mathrm{PM}_{2.5}$ 作为首要变量进行考虑, 代入 DLNM 模型进行拟合。模型拟合结果显示，深圳 及温州市的非输入确诊人数的 $\mathrm{RR}$ 与 $\mathrm{PM}_{2.5}$ 均存在较强的非线性关系。表 5 为深圳市、温州市 $\mathrm{PM}_{2.5}$ 与非输入型确诊病例的详细 $\mathrm{RR}$ 值及其置信区间。其中，以 $22.5 \mathrm{\mu g} / \mathrm{m}^{3}$ 为基准，表 5 中 各 $\mathrm{RR}$ 值表示 $\mathrm{PM}_{2.5}$ 的不同浓度与基准浓度 $\left(22.5 \mu \mathrm{g} / \mathrm{m}^{3}\right)$ 相比时的 COVID-19 的相关危险度, * 表示该滞后天数下 $\mathrm{RR}$ 值及 $\mathrm{RR}$ 的置信区间均处于 1 的同一侧，表示该处的结果较为显著。

由表 5 可知, 当 $\mathrm{PM}_{2.5}$ 的浓度为 $19 \mu \mathrm{g} / \mathrm{m}^{3}$, 滞后天数为 1-14 天时, $\mathrm{PM}_{2.5}$ 浓度的降低使深 圳市的非输入确诊人数增加的风险降低 $(\mathrm{RR}<1,95 \% \mathrm{CI}=(0,1)$; 同样浓度下, 滞后天数为 $0-2$ 天时, $\mathrm{PM}_{2.5}$ 浓度的降低使温州市的非输入确诊人数增加的风险降低 $(\mathrm{RR}<1,95 \% \mathrm{CI}=(0,1)$ 。

当 $\mathrm{PM}_{2.5}$ 的浓度为 $26 \mu \mathrm{g} / \mathrm{m}^{3}$ 且滞后天数为 3-14 天时, $\mathrm{PM}_{2.5}$ 浓度的上升使深圳市的非输 入确诊人数增加的风险增高 $(\mathrm{RR}>1,95 \% \mathrm{CI}=(1,3.9))$; 同样浓度下，当滞后天数为 $0-2$ 天时， $\mathrm{PM}_{2.5}$ 浓度的增加使温州市的 COVID-19 疫情感染几率上升（RR>1，95\%CI $=(1,3.3)$ 。

\subsubsection{CO}

文章接着考虑将 $\mathrm{CO}$ 作为主要指标放入模型。模型结果显示，仅温州市非输入确诊人数的 $\mathrm{RR}$ 与 $\mathrm{CO}$ 存在较弱的非线性关系。表 6 为深圳市与温州市 $\mathrm{CO}$ 与非输入型确诊病例的详细 $\mathrm{RR}$ 
表 4: 深圳、温州市 $\mathrm{NO}_{2}$ 与非输入确诊风险关联情况。

\begin{tabular}{|c|c|c|c|c|}
\hline \multirow{3}{*}{ 滞后天数 } & \multicolumn{2}{|c|}{$11 \mu \mathrm{g} / \mathrm{m}^{3}(\mathbf{3 0 \%})$} & \multicolumn{2}{|c|}{$23 \mu \mathrm{g} / \mathrm{m}^{3}(\mathbf{7 0 \%})$} \\
\hline & 深圳 & 温州 & 深圳 & 温州 \\
\hline & $\mathrm{RR}(95 \% \mathrm{CI})$ & $\mathrm{RR}(95 \% \mathrm{CI})$ & $\mathrm{RR}(95 \% \mathrm{CI})$ & $\mathrm{RR}(95 \% \mathrm{CI})$ \\
\hline 0 & $5.57(0.62,50.30)$ & $2.37(0.70,7.99)$ & $1.07(0.02,55.26)$ & $20.52(0.48,879.68)$ \\
\hline 1 & $5.50(1.11,27.33)^{*}$ & $1.73(0.66,4.56)$ & $1.11(0.04,30.19)$ & $11.69(0.54,253.21)$ \\
\hline 2 & $5.32(1.55,18.31)^{*}$ & $1.30(0.60,2.81)$ & $1.15(0.07,18.94)$ & $6.9(0.60,79.15)$ \\
\hline 3 & $4.97(1.51,16.38)^{*}$ & $1.01(0.53,1.94)$ & $1.16(0.10,13.91)$ & $4.37(0.66,28.89)$ \\
\hline 4 & $4.46(1.14,17.46)^{*}$ & $0.83(0.46,1.51)$ & $1.15(0.12,11.47)$ & $2.98(0.71,12.50)$ \\
\hline 5 & $3.86(0.78,19.00)$ & $0.71(0.40,1.27)$ & $1.12(0.13,9.99)$ & $2.19(0.76,6.30)$ \\
\hline 6 & $3.23(0.53,19.63)$ & $0.64(0.36,1.13)$ & $1.07(0.13,8.78)$ & $1.70(0.80,3.65)$ \\
\hline 7 & $2.63(0.37,18.86)$ & $0.59(0.34,1.03)$ & $1.01(0.14,7.56)$ & $1.41(0.83,2.39)$ \\
\hline 8 & $2.08(0.26,16.85)$ & $0.56(0.33,0.96)^{*}$ & $0.94(0.14,6.31)$ & $1.22(0.85,1.75)$ \\
\hline 9 & $1.61(0.18,14.12)$ & $0.55(0.33,0.92)^{*}$ & $0.87(0.15,5.14)$ & $1.10(0.85,1.43)$ \\
\hline 10 & $1.23(0.13,11.24)$ & $0.55(0.34,0.88)^{*}$ & $0.80(0.15,4.15)$ & $1.03(0.81,1.31)$ \\
\hline 11 & $0.92(0.10,8.63)$ & $0.56(0.36,0.87)^{*}$ & $0.72(0.15,3.41)$ & $0.99(0.74,1.33)$ \\
\hline 12 & $0.68(0.07,6.48)$ & $0.58(0.39,0.87)^{*}$ & $0.65(0.14,2.94)$ & $0.97(0.67,1.41)$ \\
\hline 13 & $0.50(0.05,4.84)$ & $0.60(0.40,0.90)^{*}$ & $0.58(0.12,2.73)$ & $0.97(0.60,1.55)$ \\
\hline 14 & $0.37(0.04,3.64)$ & $0.63(0.41,0.96)^{*}$ & $0.52(0.10,2.75)$ & $0.97(0.54,1.73)$ \\
\hline
\end{tabular}

表 5: 深圳、温州市 $\mathrm{PM}_{2.5}$ 与非输入确诊风险关联情况。

\begin{tabular}{|c|c|c|c|c|}
\hline \multirow{3}{*}{ 滞后天数 } & \multicolumn{2}{|c|}{$19 \mu \mathrm{g} / \mathrm{m}^{3}(\mathbf{3 0 \%})$} & \multicolumn{2}{|c|}{$26 \mu \mathrm{g} / \mathrm{m}^{3}(\mathbf{7 0 \%})$} \\
\hline & 深圳 & 温州 & 深圳 & 温州 \\
\hline & $\mathrm{RR}(95 \% \mathrm{CI})$ & $\mathrm{RR}(95 \% \mathrm{CI})$ & $\mathrm{RR}(95 \% \mathrm{CI})$ & $\mathrm{RR}(95 \% \mathrm{CI})$ \\
\hline 0 & $0.72(0.47,1.11)$ & $0.55(0.40,0.73)^{*}$ & $0.85(0.59,1.23)$ & $2.19(1.49,3.20)^{*}$ \\
\hline 1 & $0.58(0.40,0.84)^{*}$ & $0.61(0.46,0.80)^{*}$ & $1.06(0.77,1.45)$ & $1.97(1.34,2.91)^{*}$ \\
\hline 2 & $0.47(0.33,0.67)^{*}$ & $0.67(0.48,0.93)^{*}$ & $1.29(0.96,1.73)$ & $1.80(1.15,2.81)^{*}$ \\
\hline 3 & $0.40(0.28,0.58)^{*}$ & $0.72(0.49,1.07)$ & $1.53(1.15,2.05)^{*}$ & $1.66(0.99,2.77)$ \\
\hline 4 & $0.36(0.24,0.52)^{*}$ & $0.77(0.50,1.20)$ & $1.76(1.31,2.36)^{*}$ & $1.55(0.88,2.74)$ \\
\hline 5 & $0.33(0.22,0.49)^{*}$ & $0.81(0.50,1.31)$ & $1.96(1.45,2.65)^{*}$ & $1.47(0.80,2.69)$ \\
\hline 6 & $0.31(0.21,0.47)^{*}$ & $0.84(0.52,1.37)$ & $2.13(1.56,2.90)^{*}$ & $1.41(0.76,2.61)$ \\
\hline 7 & $0.30(0.20,0.46)^{*}$ & $0.86(0.53,1.40)$ & $2.25(1.64,3.10)^{*}$ & $1.37(0.74,2.52)$ \\
\hline 8 & $0.30(0.20,0.47)^{*}$ & $0.87(0.55,1.39)$ & $2.33(1.68,3.25)^{*}$ & $1.34(0.75,2.41)$ \\
\hline 9 & $0.31(0.20,0.48)^{*}$ & $0.88(0.57,1.35)$ & $2.37(1.68,3.36)^{*}$ & $1.32(0.77,2.28)$ \\
\hline 10 & $0.33(0.21,0.51)^{*}$ & $0.88(0.59,1.29)$ & $2.38(1.64,3.45)^{*}$ & $1.32(0.80,2.16)$ \\
\hline 11 & $0.35(0.22,0.56)^{*}$ & $0.87(0.62,1.21)$ & $2.35(1.57,3.53)^{*}$ & $1.31(0.85,2.04)$ \\
\hline 12 & $0.37(0.22,0.62)^{*}$ & $0.86(0.64,1.14)$ & $2.31(1.48,3.61)^{*}$ & $1.32(0.90,1.93)$ \\
\hline 13 & $0.40(0.23,0.70)^{*}$ & $0.84(0.66,1.07)$ & $2.25(1.37,3.70)^{*}$ & $1.33(0.95,1.85)$ \\
\hline 14 & $0.43(0.23,0.81)^{*}$ & $0.83(0.66,1.03)$ & $2.19(1.26,3.80)^{*}$ & $1.34(0.99,1.81)$ \\
\hline
\end{tabular}


表 6: 深圳、温州市 $\mathrm{CO}$ 与非输入确诊风险关联情况。

\begin{tabular}{|c|c|c|c|c|}
\hline \multirow{3}{*}{ 滞后天数 } & \multicolumn{2}{|c|}{$0.5 \mathrm{mg} / \mathrm{m}^{3}$} & \multicolumn{2}{|c|}{$0.7 \mathrm{mg} / \mathrm{m}^{3}$} \\
\hline & 深圳 & 温州 & 深圳 & 温州 \\
\hline & $\mathrm{RR}(95 \% \mathrm{CI})$ & $\mathrm{RR}(95 \% \mathrm{CI})$ & $\mathrm{RR}(95 \% \mathrm{CI})$ & $\mathrm{RR}(95 \% \mathrm{CI})$ \\
\hline 0 & $1.80(0.35,9.14)$ & $1.60(0.89,2.89)$ & $0.93(0.00,237.02)$ & $0.33(0.14,0.78)^{*}$ \\
\hline 1 & $1.74(0.29,10.36)$ & $1.45(0.80,2.63)$ & $1.26(0.01,167.95)$ & $0.42(0.20,0.86)^{*}$ \\
\hline 2 & $1.68(0.21,13.58)$ & $1.33(0.68,2.60)$ & $1.67(0.02,143.37)$ & $0.52(0.25,1.08)$ \\
\hline 3 & $1.64(0.15,17.76)$ & $1.23(0.57,2.63)$ & $2.13(0.03,141.44)$ & $0.63(0.29,1.41)$ \\
\hline 4 & $1.60(0.12,21.60)$ & $1.15(0.51,2.62)$ & $2.59(0.05,147.72)$ & $0.74(0.31,1.77)$ \\
\hline 5 & $1.57(0.10,24.08)$ & $1.09(0.47,2.53)$ & $3.01(0.06,152.22)$ & $0.83(0.33,2.08)$ \\
\hline 6 & $1.56(0.10,24.68)$ & $1.05(0.46,2.38)$ & $3.36(0.08,147.93)$ & $0.91(0.36,2.27)$ \\
\hline 7 & $1.55(0.10,23.45)$ & $1.01(0.47,2.18)$ & $3.64(0.10,132.60)$ & $0.96(0.40,2.32)$ \\
\hline 8 & $1.54(0.11,20.98)$ & $0.99(0.50,1.95)$ & $3.81(0.13,109.01)$ & $1.00(0.44,2.24)$ \\
\hline 9 & $1.54(0.13,18.01)$ & $0.97(0.55,1.73)$ & $3.90(0.18,82.75)$ & $1.01(0.49,2.10)$ \\
\hline 10 & $1.54(0.16,15.18)$ & $0.96(0.60,1.54)$ & $3.90(0.26,58.94)$ & $1.01(0.52,1.97)$ \\
\hline 11 & $1.55(0.19,12.97)$ & $0.96(0.64,1.43)$ & $3.84(0.36,40.41)$ & $0.99(0.51,1.91)$ \\
\hline 12 & $1.56(0.21,11.63)$ & $0.96(0.63,1.45)$ & $3.72(0.50,27.71)$ & $0.96(0.47,1.98)$ \\
\hline 13 & $1.57(0.22,11.33)$ & $0.96(0.57,1.61)$ & $3.58(0.64,20.09)$ & $0.93(0.39,2.20)$ \\
\hline 14 & $1.58(0.21,12.24)$ & $0.96(0.49,1.90)$ & $3.43(0.71,16.57)$ & $0.89(0.31,2.55)$ \\
\hline
\end{tabular}

值及其置信区间。其中，以 $0.6 \mathrm{mg} / \mathrm{m}^{3}$ 为基准，表 6 中各 $\mathrm{RR}$ 值表示 $\mathrm{CO}$ 不同浓度与基准浓度 $\left(0.6 \mathrm{mg} / \mathrm{m}^{3}\right)$ 相比时的 COVID-19 的相关危险度， * 表示该滞后天数下 RR 值及 RR 的置信区 间均处于 1 的同一侧，表示该处的结果较为显著。

由表 6 可知, 仅当 $\mathrm{CO}$ 的浓度为 $0.7 \mathrm{mg} / \mathrm{m}^{3}$ 且滞后天数为 $0-1$ 天时, $\mathrm{CO}$ 的浓度增加使温州 市的非输入确诊病例增加的风险降低 $(\mathrm{RR}<1,95 \% \mathrm{CI}=(0,1))$ 。

\subsection{4 $\quad \mathrm{O}_{3}$}

文章接着考虑将 $\mathrm{O}_{3}$ 作为主要指标。模型结果显示, 非输入确诊人数的 $\mathrm{RR}$ 与 $\mathrm{O}_{3}$ 存在较强 且复杂的非线性关系。表 7 为深圳市与温州市 $\mathrm{O}_{3}$ 与非输入型确诊病例的详细 $R R$ 值及其置信区 间。其中, 以 $78 \mu \mathrm{g} / \mathrm{m}^{3}$ 为基准, 表 7 中各 $\mathrm{RR}$ 值表示 $\mathrm{O}_{3}$ 不同浓度与基准浓度 $\left(78 \mu \mathrm{g} / \mathrm{m}^{3}\right)$ 相 比较时 COVID-19 的相关危险度，* 表示该滞后天数下 RR 值及 RR 的置信区间均处于 1 的同 一侧，表示该处的结果较为显著。

由表 7 可知, 当 $\mathrm{O}_{3}$ 的浓度为 $63.7 \mu \mathrm{g} / \mathrm{m}^{3}$ 时, 浓度的下降均使两座城市的非输入确诊人数增 加的风险降低 $(\mathrm{RR}<1,95 \% \mathrm{CI}=(0,1))$, 其中深圳市的滞后天数为 5-14 天，温州市的滞后天 数为 $1-14$ 天。

当 $\mathrm{O}_{3}$ 的浓度为 $92.3 \mu \mathrm{g} / \mathrm{m}^{3}$ 且滞后天数为 0-14 天时, 浓度的增加将使深圳市的非输入确 诊感染风险上升 $(\mathrm{RR}>1,95 \% \mathrm{CI}=(1,168.4))$ 。在同样的浓度下, 滞后天数为 $0-1 、 14$ 天时, 浓 度的增加使温州市非输入确诊病例增加的风险降低 $(\mathrm{RR}<1,95 \% \mathrm{CI}=(0,1))$; 而在滞后天数为 2-13 天时，浓度的增加使温州市非输入确诊病例增加的风险增加（RR $>1 ， 95 \% \mathrm{CI}=(1,25.3)$ 。 由于在大部分位置, $\mathrm{O}_{3}$ 指标的 $\mathrm{RR}$ 结果均较为显著 ( $R R$ 的置信区间位于 1 的同一侧), 现 
表 7: 深圳、温州市 $\mathrm{O}_{3}$ 与非输入确诊风险关联情况。

\begin{tabular}{|c|c|c|c|c|}
\hline \multirow{3}{*}{ 滞后天数 } & \multicolumn{2}{|c|}{$63.7 \mu \mathrm{g} / \mathrm{m}^{3}(\mathbf{3 0 \%})$} & \multicolumn{2}{|c|}{$92.3 \mu \mathrm{g} / \mathrm{m}^{3}(\mathbf{7 0 \%})$} \\
\hline & 深圳 & 温州 & 深圳 & 温州 \\
\hline & $\mathrm{RR}(95 \% \mathrm{CI})$ & $\mathrm{RR}(95 \% \mathrm{CI})$ & $\mathrm{RR}(95 \% \mathrm{CI})$ & $\mathrm{RR}(95 \% \mathrm{CI})$ \\
\hline 0 & $0.59(0.19,1.84)$ & $4.01(3.2,5.01)^{*}$ & $1.90(1.08,3.35)^{*}$ & $0.18(0.12,0.28)^{*}$ \\
\hline 1 & $0.48(0.16,1.49)$ & $0.58(0.54,0.63)^{*}$ & $4.18(1.92,9.08)^{*}$ & $0.65(0.52,0.82)^{*}$ \\
\hline 2 & $0.40(0.13,1.27)$ & $0.10(0.08,0.14)^{*}$ & $8.51(2.93,24.7)^{*}$ & $2.06(1.82,2.33)^{*}$ \\
\hline 3 & $0.34(0.11,1.13)$ & $0.03(0.02,0.04)^{*}$ & $14.94(4.00,55.81)^{*}$ & $5.10(4.30,6.06)^{*}$ \\
\hline 4 & $0.31(0.09,1.03)$ & $0.01(0.01,0.02)^{*}$ & $22.28(4.98,99.79)^{*}$ & $9.74(7.56,12.54)^{*}$ \\
\hline 5 & $0.29(0.09,0.96)^{*}$ & $0.00(<0.01)^{*}$ & $28.64(5.73,143.23)^{*}$ & $14.64(10.71,20)^{*}$ \\
\hline 6 & $0.28(0.09,0.90)^{*}$ & $0.00(<0.01)^{*}$ & $32.18(6.15,168.37)^{*}$ & $17.74(12.56,25.05)^{*}$ \\
\hline 7 & $0.28(0.09,0.85)^{*}$ & $0.00(<0.01)^{*}$ & $32.11(6.22,165.74)^{*}$ & $17.76(12.49,25.25)^{*}$ \\
\hline 8 & $0.29(0.10,0.81)^{*}$ & $0.00(<0.01)^{*}$ & $28.87(5.96,139.85)^{*}$ & $15.05(10.76,21.05)^{*}$ \\
\hline 9 & $0.31(0.12,0.77)^{*}$ & $0.01(<0.01)^{*}$ & $23.73(5.43,103.66)^{*}$ & $11.05(8.19,14.90)^{*}$ \\
\hline 10 & $0.33(0.15,0.74)^{*}$ & $0.01(0.01,0.02)^{*}$ & $18.11(4.74,69.26)^{*}$ & $7.20(5.63,9.20)^{*}$ \\
\hline 11 & $0.36(0.18,0.72)^{*}$ & $0.03(0.02,0.05)^{*}$ & $13.02(3.95,42.90)^{*}$ & $4.26(3.56,5.10)^{*}$ \\
\hline 12 & $0.40(0.22,0.72)^{*}$ & $0.07(0.05,0.10)^{*}$ & $8.96(3.15,25.44)^{*}$ & $2.35(2.12,2.61)^{*}$ \\
\hline 13 & $0.44(0.26,0.75)^{*}$ & $0.18(0.14,0.23)^{*}$ & $5.98(2.38,14.99)^{*}$ & $1.23(1.20,1.28)^{*}$ \\
\hline 14 & $0.49(0.30,0.82)^{*}$ & $0.49(0.44,0.55)^{*}$ & $3.93(1.69,9.15)^{*}$ & $0.63(0.59,0.69)^{*}$ \\
\hline
\end{tabular}

绘制 $\mathrm{O}_{3}$ 的 $\mathrm{RR}$ 指标三维图，以整体观察 $\mathrm{O}_{3}$ 对疫情影响的趋势，如图3所示。

在图3所示的三维图中，横向的 $x$ 轴表示 $\mathrm{O}_{3}$ 的浓度范围，纵向的 $y$ 轴表示滞后天数 (0-14 天)，竖向的 $z$ 轴表示 $\mathrm{RR}$ 取值。可以看出，深圳和温州市 2 座城市内的 $\mathrm{O}_{3}$ 大体影响趋势类似， 表现为, $\mathrm{O}_{3}$ 浓度越高, COVID-19 疫情风险越大; 从滞后天数来看, 在滞后 7 天左右时 $\mathrm{RR}$ 达 到最大，随后向两边逐渐下降。

对气象因素和空气质量因素的分析中，我们选取了各指标的中位数作为基准水平，分别对各 指标数据范围从 10\% 到 $90 \%$ ，每间隔 10\% 的数据与对应的基准水平相比较的 COVID-19 的非 输入确诊人数进行相对危险分析。我们在各指标对应的基准水平低或高的数据范围内, 各截取一 个比例呈现于正文中。

\section{4 讨论}

本文获取了深圳市和温州市内 2020 年 1 月 1 日至 2 月 29 日（60 天）包括平均温度、平 均日平均相对湿度、日平均风速、 $\mathrm{NO}_{2} 、 \mathrm{PM}_{2.5} 、 \mathrm{CO}$ 和 $\mathrm{O}_{3}$ 的数据, 以及上述 2 个城市内截止 2 月 29 日的确诊、死亡、治愈、非输入型 COVID-19 确诊病例数据。文章对深圳市和温州市内 的气象数据、空气质量及 COVID-19 确诊数据进行了流行病学特征描述及相关性探索分析, 发 现气象和大气污染中的诸多因素与非输入确诊人数存在滞后非线性相关性，这与过往的对呼吸 疾病的研究相吻合张江华等 (2014)。

我们用分布滞后非线性模型拟合了气象因素和空气质量共 7 个指标与 COVID-19 在深圳市 和温州市之间的非线性以及滞后效应。本研究发现，深圳市和温州市在 7 个指标与 COVID-19 
深圳

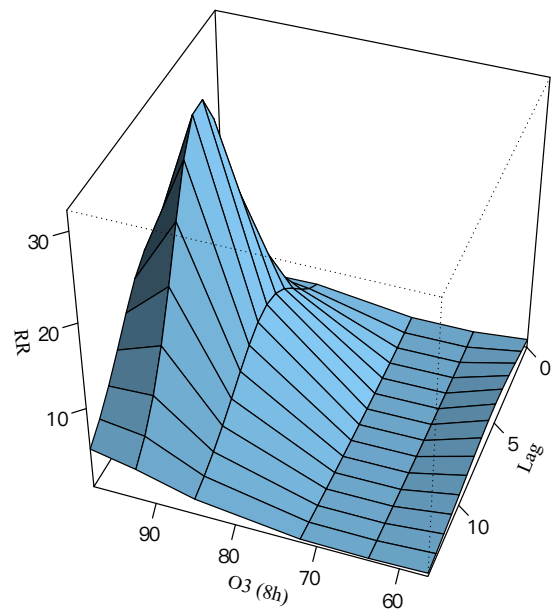

温州

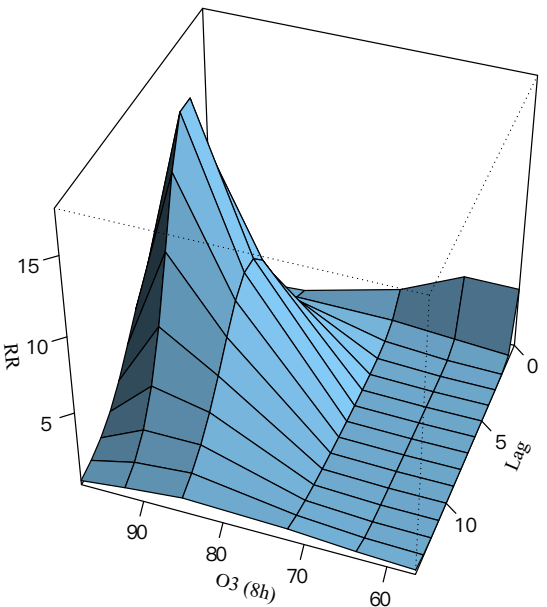

图 3: 深圳、温州市 $\mathrm{O}_{3}$ 三维 $\mathrm{RR}$ 关系图。

的效应关系上呈现明显的差异。但所有指标在两个城市间，都与 COVID-19 的患病人数呈现非 线性关系Yongjian et al. (2020)。

本研究结果显示, 以日平均气温 $15^{\circ} \mathrm{C}$ 为基准时, 当日平均温度 $\left(11^{\circ} \mathrm{C}\right.$ ) 低于这个基准温度 时，温州市的 COVID-19 的非输入型日患病人数与温度变化有显著相反关系，在滞后 1-3 天后 患病人数显著增加，即低温提升了疫情的传播的风险Xie et al. (2020)。以日平均相对湿度 $76 \%$ 为基准时，当日平均相对湿度低于或高于这个基准相对湿度时，温州市的 COVID-19 的非输入 确诊人数与相对湿度有显著正向关系。即当相对湿度降低时, COVID-19 的疫情的传播风险会降 低，反之，会升高。有文献指出Auler et al. (2020), COVID-19 的疫情的发展会在相对湿度为 $80 \%$ 时发展迅速。以日平均风速 $7.25 \mathrm{~km} / \mathrm{h}$ 为基准时，当日平均风速低于该基准日平均风速时， 滞后 2-14 天的温州市的非输入确诊人数显著下降，当日平均风速高于该基准日平均风速时，深 圳市的非输入确诊人数显著下降。也就是当日平均风速处在基准平均速度时会加速 COVID-19 疫情的传播Tosepu et al. (2020)。

另一方面, 深圳市和温州市的 COVID-19 的非输入型日患病人数都与 $\mathrm{NO}_{2}$ 呈现显著的非 线性关系，且具有显著的不同时间的滞后效应。以 $\mathrm{NO}_{2}$ 为 $16 \mu \mathrm{g} / \mathrm{m}^{3}$ 为基准浓度时，当 $\mathrm{NO}_{2}$ 在空气中浓度降低时，会促进 COVID-19 的患病人数的增长。以 $\mathrm{PM}_{2.5}$ 为 $22.5 \mu \mathrm{g} / \mathrm{m}^{3}$ 为基准 浓度时, 当 $\mathrm{PM}_{2.5}$ 在空气中浓度增加或者降低时, 温州市的 COVID-19 的非输入型确诊人数都 与 $\mathrm{PM}_{2.5}$ 呈现显著的非线性关系，且具有显著的较长时间（>8 天）的滞后效应。而深圳市的 COVID-19 的非输入型日患病人数与 $\mathrm{PM}_{2.5}$ 在浓度增加时，呈现显著的非线性与滞后效应。当 $\mathrm{PM}_{2.5}$ 浓度增加时，会增加 COVID-19 的患病人数Chen et al. (2020); Yongjian et al. (2020); Tian et al. (2020)。同样以 $\mathrm{O}_{3}$ 为 $78 \mu \mathrm{g} / \mathrm{m}^{3}$ 为基准浓度时，当 $\mathrm{O}_{3}$ 在空气中浓度增加或者降低 时, 深圳市和温州市的 COVID-19 的非输入型日患病人数都与 $\mathrm{O}_{3}$ 呈现显著的非线性关系，且 
具有显著的不同时间的滞后效应。

此外, 有研究表明Feng et al. (2020), 空气因素 (例如风速和相对湿度) 会影响飞沫在空气中 的传播，而当受感染人群咳嗽或者打喷德时，包裹着 SARS-CoV-2 飞沫，会随着相对湿度的增强 而变大。并随着风速的加强，会飞散更远的距离。同时由于病毒具有潜伏期Backer et al. (2020); Lauer et al. (2020), 所以可能会表现为一定的滞后效应。因而，保持严格的社交距离，是避免二 代感染的有效手段。

本文探究了多种气象因素与空气质量因素对 COVID-19 的传播以及非输入患病人数的影响。 根据深圳市与温州市的数据探索发现, 日平均温度、日平均相对湿度、和日平均风速、 $\mathrm{NO}_{2} 、 \mathrm{PM}_{2.5}$ 、 和 $\mathrm{O}_{3}$ 这六个指标与 COVID-19 的非输入患病人数存在显著效应。其中日平均相对湿度、 $\mathrm{PM}_{2.5}$ 与 COVID-19 患病人数呈显著正相关关系。日平均风速、 $\mathrm{NO}_{2}$ 与 $\mathrm{O}_{3}$ 在不同城市对患病人数的 影响有所差异, 并且不同地区的相关性与滞后效应有所不同。文章为不同地区的空气与气象因素 对 COVID-19 的分析提供了新的思路，有助于疫情分析与防控，且具有一定的参考价值。

\section{补充资料}

本文分析所需数据和 R 代码以及附录在期刊网页存档。附录包括 A. 气象因素、大气污染 因素折线图总览; B. 深圳市与温州市各个模型的离散参数估计结果; C. 气象因素、大气污染因素 模型拟合情况总览; D. 深圳市、温州市的气象与大气污染因素与非输入确诊风险关联情况表。

\section{参考文献}

中华人民共和国国家卫生健康委员会, 2020. 新型冠状病毒肺炎诊疗方案（试行第七版). http: //www.nhc.gov.cn/xcs/zhengcwj/202003/. http://www.nhc.gov.cn/xcs/zhengcwj/202003/. 宋捷, 2015. 大气污染和气象因素对传染病的交互影响. 兰州: 兰州大学.

张江华, 郭常义, 许慧慧, 李源培, 张莉君, 东春阳, 施烨闻, 钱海雷, 金奇昂, 2014. 上海市大气污 染与某医院呼吸系统疾病门诊量关系的时间序列研究. 环境与职业医学, 31(11): 846-851.

李青春, 陆晨, 刘彦, 戴丽萍, 1999. 北京地区呼吸道疾病与气象条件关系的分析. 气象, 25(3): 3-5.

杨军, 欧春泉, 丁研, 陈平雁, 2012. 分布滞后非线性模型. 中国卫生统计, 29(5): 772-773.

段振华, 高绪芳, 杜慧兰, 覃芳葵, 陈俊, 鞠勇, 伍霞, 胡燕, 2015. 成都市空气 PM2. 5 浓度与呼吸 系统疾病门诊人次的时间序列研究. 现代预防医学, 42(4): 611-614.

王艳琴, 2012. 环境保护部发布 HJ633-2012《环境空气质量指数 (AQI) 技术规定 (试行)》. 中 国标准导报(4): 49.

王铮, 蔡砥, 李山, 郑一萍, 王荣, 吴兵, 黎华群, 陈建国, 等, 2003. 中国 SARS 流行的季节性风险 探讨. 地理研究, 22(5): 541-550.

谷少华, 贺天锋, 陆蓓蓓, 徐倩倩, 梅秋红, 张思恒, 2016. 基于分布滞后非线性模型的归因风险评

估方法及应用. 中国卫生统计, 33(6): 959-962.

路凤, 李亚伟, 李成橙, 陈晨, 宋士勋, 郭玉明, 施小明, 2018. 时间序列分析在空气污染与健康领 域的应用及其 R 软件实现. 中国卫生统计, 35(4): 622-625. 
靳英辉, 蔡林, 程真顺, 程虹, 邓通, 范逸品, 等, 2020. 新型冠状病毒 (2019-nCoV) 感染的肺炎诊 疗快速建议指南 (标准版). 解放军医学杂志, 45(1): 1-20.

Anderson BG, Bell ML, 2009. Weather-related mortality: How heat, cold, and heat waves affect mortality in the United States. Epidemiology (Cambridge, Mass.), 20(2): 205-213.

Armstrong B, 2006. Models for the relationship between ambient temperature and daily mortality. Epidemiology, 17(6): 624-631.

Auler AC, Cássaro F, da Silva VO, Pires LF, 2020. Evidence that high temperatures and intermediate relative humidity might favor the spread of COVID-19 in tropical climate: A case study for the most affected Brazilian cities. Science of The Total Environment, 729: 139090.

Backer JA, Klinkenberg D, Wallinga J, 2020. Incubation period of 2019 novel coronavirus (2019$\mathrm{nCoV}$ ) infections among travellers from Wuhan, China, 20-28 January 2020. Eurosurveillance, 25(5): 2000062.

Chen K, Wang M, Huang C, Kinney PL, Anastas PT, 2020. Air pollution reduction and mortality benefit during the COVID-19 outbreak in China. The Lancet Planetary Health, 4(6): e210$\mathrm{e} 212$.

Ciencewicki J, Jaspers I, 2007. Air pollution and respiratory viral infection. Inhalation Toxicology, 19(14): 1135-1146.

Feng Y, Marchal T, Sperry T, Yi H, 2020. Influence of wind and relative humidity on the social distancing effectiveness to prevent COVID-19 airborne transmission: A numerical study. Journal of Aerosol Science, 147: 105585.

Gasparrini A, 2011. Distributed lag linear and non-linear models in R: The package dlnm. Journal of Statistical Software, 43(8): 1-20. https://europepmc.org/articles/PMC3191524.

Gasparrini A, Armstrong B, 2013. Reducing and meta-analysing estimates from distributed lag non-linear models. BMC Medical Research Methodology, 13(1): 1-10.

Gasparrini A, Armstrong B, Kenward MG, 2010. Distributed lag non-linear models. Statistics in Medicine, 29(21): 2224-2234.

Gasparrini A, Guo Y, Hashizume M, Lavigne E, Zanobetti A, Schwartz J, Tobias A, Tong S, Rocklöv J, Forsberg B, et al., 2015. Mortality risk attributable to high and low ambient temperature: A multicountry observational study. The Lancet, 386(9991): 369-375.

Hanna AF, Yeatts KB, Xiu A, Zhu Z, Smith RL, Davis NN, Talgo KD, Arora G, Robinson PJ, Meng Q, et al., 2011. Associations between ozone and morbidity using the Spatial Synoptic Classification system. Environmental Health, 10(1): 49.

Knott GD, 2000. Interpolating cubic splines: volume 18. Springer Science \& Business Media.

Lauer SA, Grantz KH, Bi Q, Jones FK, Zheng Q, Meredith HR, Azman AS, Reich NG, Lessler J, 2020. The incubation period of coronavirus disease 2019 (COVID-19) from publicly reported confirmed cases: Estimation and application. Annals of Internal Medicine, 172(9): 577-582.

Li Q, Guan X, Wu P, Wang X, Zhou L, Tong Y, Ren R, Leung KS, Lau EH, Wong JY, et al., 2020. 
Early transmission dynamics in Wuhan, China, of novel coronavirus-infected pneumonia. New England Journal of Medicine, 382(13): 1199-1207.

Martelletti L, Martelletti P, 2020. Air pollution and the novel Covid-19 disease: A putative disease risk factor. SN Comprehensive Clinical Medicine, 2: 383-387.

McMichael AJ, Wilkinson P, Kovats RS, Pattenden S, Hajat S, Armstrong B, Vajanapoom N, Niciu EM, Mahomed H, Kingkeow C, et al., 2008. International study of temperature, heat and urban mortality: The 'ISOTHURM' project. International Journal of Epidemiology, 37(5): 1121-1131.

Runkle JD, Sugg MM, Leeper RD, Rao Y, Mathews JL, Rennie JJ, 2020. Short-term effects of weather parameters on COVID-19 morbidity in select US cities. Science of The Total Environment, 740: 140093.

Sohrabi C, Alsafi Z, O’ Neill N, Khan M, Kerwan A, Al-Jabir A, Iosifidis C, Agha R, 2020. World Health Organization declares global emergency: A review of the 2019 novel coronavirus (COVID-19). International Journal of Surgery, 76: 71-76.

The Weather Company, an IBM business, 2020. Weather underground. https://www. wunderground.com/. https://www.wunderground.com/.

Tian T, Zhang J, Hu L, Jiang Y, Duan C, Wang X, Zhang H, 2020. Risk factors associated with mortality of COVID-19 in 3125 counties of the United States. medRxiv Preprint. https: //www.medrxiv.org/content/10.1101/2020.05.18.20105544v4.

Tosepu R, Gunawan J, Effendy DS, Lestari H, Bahar H, Asfian P, et al., 2020. Correlation between weather and Covid-19 pandemic in Jakarta, Indonesia. Science of The Total Environment, 725: 138436.

Vanos JK, Cakmak S, Kalkstein LS, Yagouti A, 2015. Association of weather and air pollution interactions on daily mortality in 12 Canadian cities. Air Quality, Atmosphere \& Health, 8 (3): 307-320.

Wang M, Jiang A, Gong L, Luo L, Guo W, Li C, Zheng J, Li C, Yang B, Zeng J, et al., 2020. Temperature significant change COVID-19 transmission in 429 cities. Cold Spring Harbor Laboratory Press. MedRxiv Preprint. https://www.medrxiv.org/content/10.1101/2020.02. $22.20025791 \mathrm{v} 1$.

Xie J, Zhu Y, 2020. Association between ambient temperature and COVID-19 infection in 122 cities from China. Science of The Total Environment, 724: 138201.

Yongjian Z, Jingu X, Fengming H, Liqing C, 2020. Association between short-term exposure to air pollution and covid-19 infection: Evidence from china. Science of the Total Environment, 727: 138704 . 